Proceedings of the 16th Czech and Slovak Conference on Magnetism, Košice, Slovakia, June 13-17, 2016

\title{
Ground-State Spin of Hubbard Ladder Model with Infinite Electron Repulsion
}

\author{
V.O. Cheranovskit ${ }^{a} *$, E.V. Ezerskaya ${ }^{a}$, D.J. Klein ${ }^{b}$ And V.V. TOKARev $^{a}$ \\ ${ }^{a}$ V.N. Karazin Kharkiv National University, Svoboda Sq., 4, 61022 Kharkiv, Ukraine \\ ${ }^{b}$ Texas A\&M University at Galveston, Galveston, TX, 77554 USA
}

\begin{abstract}
We apply perturbation theory and cyclic spin permutation formalism to study the lowest energy states of the infinite-repulsion Hubbard model on $n$-leg ladders with alternating values of one-site energies $\alpha_{i}$ for neighboring rungs. We establish the "ferromagnetic" character of ladder ground-state at electron densities in the interval $1-$ $(2 n)^{-1} \leq \rho \leq 1$ and sufficiently large alternation of one-site energies of neighbor rungs of the ladder. We also show the stability of this state against the small deviations of the values of $\alpha_{i}$ in contrast to the case of two-leg ladder formed by weakly interacting neighbor rungs with equal one-site energies.
\end{abstract}

DOI: 10.12693/APhysPolA.131.916

PACS/topics: 71.10.Fd, 75.10.Jm

\section{Introduction}

One of the simplest approaches to the study of magnetism of itinerant electrons is the Hubbard model with infinite electron repulsion (IRM). According to the Nagaoka-Thouless theorem [1, 2], for some type of lattices, the ground state of the one-band IRM with the one hole in a half-filled band corresponds to the maximal value of total spin. The Nagaoka-Thouless possibility of saturated ferromagnetism for several holes in the band is a delicate issue depending on the lattice structure and the density of electrons. In our previous works we have shown that for IRM on rectangular lattice strip formed by weakly interacting $n$-site segments (anisotropic $n$-leg ladder), there is a cascade of concentration transitions with regular oscillation of the ground-state spin between minimal and maximal values due to the creation of magnetic polarons [3, 4]. Recently "ferromagnetic" ground state was found numerically for this model on isotropic $n$-leg ladder with the density of electrons per site $\rho$ in the range $0.8 \leq \rho<1[5]$.

Our work here is devoted to the study of the ground state and lowest excitations of IRM on the $n$-leg ladder with an alternating value of one-site energies $\alpha_{i}$ for neighboring rungs. For example, $\alpha_{i}=0$ and $\alpha_{i}=\alpha$ for odd and even rungs, respectively. Here we show that at sufficiently large value of $\alpha$ and $\rho \geq 1-(2 n)^{-1}$ the ladder has the ground state with maximal value of total spin (i.e. a "ferromagnetic" ground state). Also, we find a greater relative stability as the interactions between segments deviate from the $\alpha=0$ model considered in $[3,4]$.

The difference in one site energies $\alpha_{i}$ can presumably be attained by changes in chemical structure of the ligands, and we believe that our study can be useful to the design of new nanostructured ferromagnetic materi-

*corresponding author; e-mail: cheranovskii@i.ua als based on polymeric complex compounds of transition metals.

\section{Effective spin Hamiltonians}

Consider the $n$-leg ladder formed by $L$ rungs (with the 3-leg ladder shown in Fig. 1) and described by the following IRM Hamiltonian:

$$
\begin{aligned}
\widehat{H} & =\widehat{P}\left\{\widehat{H}_{0}+t_{2} \widehat{H}_{1}\right\} \widehat{P}, \\
\widehat{H}_{0} & =\sum_{i=1}^{L} \alpha_{i} \sum_{j=1}^{n} \sum_{\sigma} \widehat{a}_{i, j, \sigma}^{\dagger} \widehat{a}_{i, j, \sigma} \\
& +t_{1} \sum_{i=1}^{L} \sum_{j=1}^{n-1} \sum_{\sigma}\left(\widehat{a}_{i, j, \sigma}^{\dagger} \widehat{a}_{i, j+1, \sigma}+\text { H.c. }\right), \\
\widehat{H}_{1} & =\sum_{i=1}^{L-1} \sum_{j=1}^{n} \sum_{\sigma}\left(\widehat{a}_{i, j, \sigma}^{\dagger} \widehat{a}_{i+1, j, \sigma}+\text { H.c. }\right),
\end{aligned}
$$

where $\widehat{P}$ is a projector into the space without doubly occupied lattice sites; $\alpha_{i}=\alpha>0$ for even rungs and $\alpha_{i}=0$ for odd rungs; $t_{1}$ is a matrix element of the electron transfer between neighboring sites of the same rung, and $t_{2}$ is for electron hops between corresponding sites in neighboring rungs.

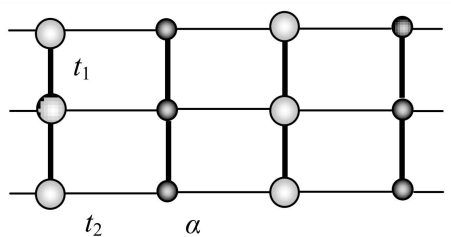

Fig. 1. Fragment of rectangular lattice strip formed by three-site segments with alternating values of one-site energies.

Let us consider the ladder formed by an even number $L$ of weakly interacting rungs (with $t_{2} \rightarrow 0$ ). For a total number of electrons $N_{e}=n L-L / 2$, the ground state of 
the projected lattice Hamiltonian (1) corresponds to the following electron distribution: each even rung is occupied by $n-1$ electrons and each odd rung is occupied by $n$ electrons. At $t_{2}=0$ this state is degenerate with respect to the configuration of spin variables $\sigma$. Weak interaction between neighboring rungs removes this degeneracy in second order of $\mathrm{PT}$ in $t_{2}$. As a result, the effective Hamiltonian describing low energy levels of the ladder is a sum of terms describing the interactions between pairs of neighboring rungs. This Hamiltonian can be derived by cyclic spin permutation technique [4]. For example, in case of two-leg ladder, formed by $L$ weakly interacting rungs, it has the form

$$
\begin{aligned}
\widehat{H}_{\mathrm{eff}} & =\sum_{l=1}^{L-1} \widehat{H}_{l, l+1}, \\
\widehat{H}_{l, l+1} & =J_{1}\left(\widehat{Q}_{k, k+2}+\widehat{Q}_{k, k+2}^{\dagger}\right)+J_{2}, \\
k & = \begin{cases}(3 l-1) / 2, & l \text { odd } \\
3 l / 2, & l \text { even }\end{cases} \\
J_{1} & =t_{1}\left(t_{2}\right)^{2} /\left[2 \alpha\left(\alpha-2 t_{1}\right)\right], \\
J_{2} & =-\left(\alpha-t_{1}\right)\left(t_{2}\right)^{2} /\left[\alpha\left(\alpha-2 t_{1}\right)\right],
\end{aligned}
$$

where $\widehat{Q}_{i, i+2}$ is an operator of cyclic permutation of three spin variables

$$
\widehat{Q}_{i, i+2}\left|\sigma_{1}, \sigma_{2}, \sigma_{3}\right\rangle=\left|\sigma_{2}, \sigma_{3}, \sigma_{1}\right\rangle \text {. }
$$

According to the extended Nagaoka theorem [1], each of these terms has the maximal value of the ground state spin. The component of "ferromagnetic" state of the ladder Hamiltonian (1) with the maximal value of the $z$-projection of total spin ("max- $S$ " state) corresponds to minimal interaction energy for each pair of neighbor rungs. Therefore, at $\alpha \gg\left|t_{2}\right|$ due to the scalar character of the Hamiltonian (1) the ladder ground state corresponds to the maximal value of total spin $S_{0}=(n L-L / 2) / 2$ at electron density $\rho=1-(2 n)^{-1}$.

\section{Stability of "ferromagnetic" ground state}

Let us consider the 2-leg ladder fragment with one "impurity" rung having the positive one-site energy $\bar{\alpha}>\alpha$ with total number of electrons $N_{e}=3 L / 2$ ( $L$ is even). For $\left|t_{1}\right| \gg\left|t_{2}\right|$ the lowest energy of the fragment corresponds either: to an electron distribution with empty "impurity" rung for $\bar{\alpha}-\alpha>2\left|t_{1}\right|$; or to a distribution with one electron on an "impurity" rung in case of $\bar{\alpha}-\alpha<2\left|t_{1}\right|$. It can be shown that neighbor rungs interact at second order of $\mathrm{PT}$ in $t_{2}$ to give an effective "antiferromagnetic" coupling of an empty rung with neighbor rungs and a "ferromagnetic" coupling for neighbor rungs with one and two electrons. This means that a 2-leg ladder with some "impurity" rungs at electron density $\rho=3 / 4$ has a ground state with the maximal value of total spin if $\left|t_{2}\right|<<\bar{\alpha}-\alpha<2\left|t_{1}\right|$.

As a check on these ideas, we performed an exact diagonalization study of the lowest energy states of the
Hamiltonian (1) for ladder fragment with $n=2, L=4$, $N_{e}=6, \alpha=\left|t_{1}\right|=1$ and $\left|t_{2}\right|=0.1$ at different values of $\Delta \alpha=\bar{\alpha}-\alpha$. The results of this study are given in Fig. 2, and turn out to neatly agree with the preceding analysis.

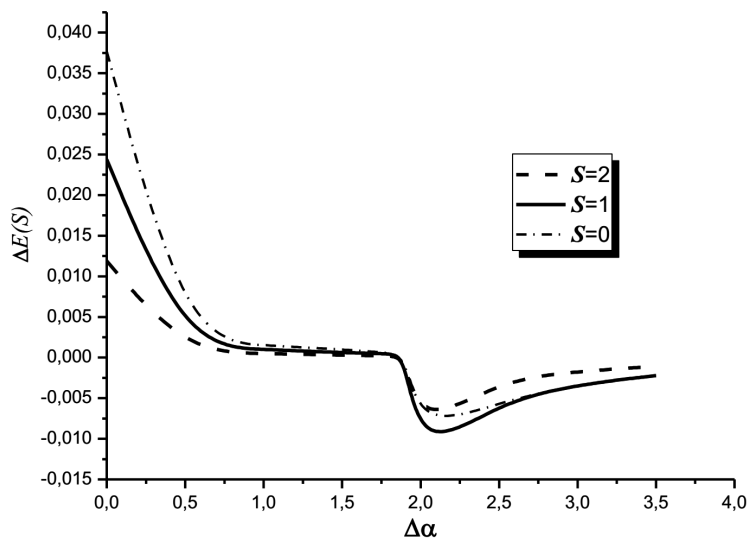

Fig. 2. The lowest energies $\Delta E(S)=E_{\min }(S)-$ $E_{\text {min }}(3)$ with given value of total spin $S$ of the ladder fragment at different values of $\Delta \alpha$.

Let us suppose that the finite fragment of the $n$-leg ladder with alternating values of one-site energies has some "impurity" rungs with $\bar{\alpha} \neq \alpha$ and total number of electrons $N_{e}=(2 n-1) L / 2$. According to PT analysis, this fragment should have a "ferromagnetic" ground state, if $4\left|t_{2}\right| \cos (\pi /(n+1))>|\bar{\alpha}-\alpha| \gg\left|t_{2}\right|$. In other words, this ground state is stable against weak changes in onesite energies. The reason for this stability is the local character of the interactions between rungs of the above ladder.

In contrast, the ground state of anisotropic ladders with the same values of one-site energies has a polaronic character at $1-(2 n)^{-1} \leq \rho<1[3,4]$, and an appearance of similar "impurity" rungs may destroy the ferromagnetic spin ordering. Thus, for the ladder fragment with $n=2, L=4, N_{e}=6, \Delta \alpha=\left|t_{1}\right|=1$ and $\left|t_{2}\right|=0.1$, an exact diagonalization study reveals a ground state spin $S_{0}=0$ at $\alpha=0$ and $S_{0}=3$ at $\alpha=1$.

In order to study the effect of donor doping on the ground-state spin of our ladder model, consider a finite fragment of $n$-leg ladder formed by an even number of rungs with $\alpha \gg\left|t_{2}\right|$ and a total number of electrons $N_{e}=(2 n-1) L / 2+1$. In this case, there are three neighboring rungs with $3 n-1$ electrons. According to [6] the ground-state spin of this electron system should take a maximal value. In $\mathrm{PT}$ at second order in $t_{2}$ all other interactions are carried out between pairs of neighboring rungs with non-equal electron fillings. These interactions have a "ferromagnetic" character, too. Thence the ladder has a maximal value of ground-state spin. Consequently, the $n$-leg ladder has a "ferromagnetic" ground state for electron densities in the interval $1-(2 n)^{-1} \leq \rho<1$.

In order to study the effect of acceptor doping on the ground-state spin of our ladder model, consider the fi- 
nite 2-leg ladder fragment with $N_{e}=3 L / 2-1$. In the $t_{2} \rightarrow 0$ limit, this fragment has a pair of neighbor rungs with two electrons. For such a pair each rung is occupied by one electron at $\alpha<2\left|t_{1}\right|$, and we have one empty rung \& neighbor rung with two electrons at $\alpha>2\left|t_{1}\right|$. These pairs of neighbor rungs interact in second PT order in $t_{2}$. To describe this interaction, it is convenient to use cyclic spin permutation techniques for the direct consideration of the electron hopping processes between neighboring lattice sites [3, 4]. The corresponding effective spin Hamiltonians are presented as

$$
\begin{aligned}
& \widehat{H}_{\text {eff }}=R_{1} \widehat{Q}_{1,2}-R_{2} \widehat{I}, \quad \text { where } \\
& \begin{cases}R_{1}=R_{2}=-2\left|t_{1}\right| / G, & \text { for } \alpha<2\left|t_{1}\right| \\
R_{1}=4\left(t_{1}\right)^{2} / G, R_{2}=2 \alpha^{2} / G-R_{1}, & \text { for } \alpha>2\left|t_{1}\right|\end{cases} \\
& G=\left(\alpha^{2}-4 t_{1}^{2}\right) / t_{2}^{2},
\end{aligned}
$$

where $\widehat{Q}_{1,2}$ is an operator of transposition of two spin variables.

For $\alpha=2\left|t_{1}\right|$ two neighbor rungs occupied by two electrons interact in first order of $\mathrm{PT}$ in $t_{2}$ whence this interaction is described by an effective Hamiltonian

$$
\widehat{H}_{\mathrm{eff}}=\left(\begin{array}{cc}
0 & \widehat{A} \\
\widehat{A} & 0
\end{array}\right), \quad \widehat{A}=\frac{t_{2}}{2}\left(\widehat{I}-\widehat{Q}_{1,2}\right) .
$$

It can be shown that the ground states of the Hamiltonians (2) and (3) correspond to minimal value of total spin $S_{0}=0$. As a result, the ground-state spin of the above ladder fragment is determined by the competition of the "ferromagnetic" and "antiferromagnetic" interactions of neighbor rungs. Similar consideration can be given for infinite 2-leg ladder with electron density $\rho<0.75$ and we may conclude that the "ferromagnetic" ground state of the anisotropic 2-leg ladder with alternating values of one-site energies may be destroyed by acceptor doping.

\section{Conclusions}

We have studied the ground state and lowest excitations of IRMs on $n$-leg ladders with alternating value of one-site energies for neighboring rungs by means of $\mathrm{PT}$ in the inter-rung parameter $t_{2}$. At electron density $1-(2 n)^{-1} \leq \rho \leq 1$ and $\alpha \gg\left|t_{2}\right|$ we have found the "ferromagnetic" ground state of the ladder to be stable. Further, we have found a stability of this state against the small deviations of the values for one-site energies. For a similar anisotropic $n$-leg ladder with equal one-site energies these deviations may destroy "ferromagnetic" ordering in the ground state.

A general point underlying this work is that electron permutations restricted to avoid double occupancies can lead to novel behaviors, which even now are not yet fully explored.

\section{Acknowledgments}

D.J.K. acknowledges the support of the Welch Foundation of Houston, Texas (via grant BD-0894).

\section{References}

[1] Y. Nagaoka, Phys. Rev. 147, 392 (1966).

[2] D.J. Thouless, Proc. Phys. Soc. Lond. 86, 893 (1965).

[3] V.Ya. Krivnov, A.A. Ovchinnikov, V.O. Cheranovskii, Synth. Met. 33, 65 (1989).

[4] V.O.Cheranovskii, O. Esenturk, H.O. Pamuk, Phys. Rev. B 58, 12260 (1998).

[5] L. Liu, H. Yao, E. Berg, S.R. White, S.A. Kivelson, Phys. Rev. Lett. 108, 126406 (2012).

[6] H. Tasaki, Phys. Rev. B 40, 9192 (1989). 